\title{
BMJ Open Treatments and outcomes of untreated cerebral cavernous malformations in China: study protocol of a nationwide multicentre prospective cohort study
}

\author{
Fuxin Lin, ${ }^{1,2}$ Qiu He, ${ }^{1,2}$ Zhuyu Gao, ${ }^{1,2}$ Lianghong Yu, ${ }^{1,2}$ Dengliang Wang, ${ }^{1,2}$ \\ Shufa Zheng, ${ }^{1,2}$ Yuanxiang Lin, ${ }^{1,2}$ Dezhi Kang (I) ${ }^{1,2}$
}

To cite: Lin F, He Q, Gao Z, et al. Treatments and outcomes of untreated cerebral cavernous malformations in China: study protocol of a nationwide multicentre prospective cohort study. BMJ Open 2020;10:e037957. doi:10.1136/ bmjopen-2020-037957

- Prepublication history for this paper is available online. To view these files, please visit the journal online (http://dx.doi org/10.1136/bmjopen-2020037957).

FL, QH and ZG contributed equally.

$\mathrm{QH}$ and $\mathrm{ZG}$ are joint first authors. $\mathrm{QH}$ and $\mathrm{ZG}$ are joint senior authors.

Received 22 February 2020 Revised 13 July 2020

Accepted 09 September 2020

Check for updates

(C) Author(s) (or their employer(s)) 2020. Re-use permitted under CC BY-NC. No commercial re-use. See rights and permissions. Published by BMJ.

${ }^{1}$ Department of Neurosurgery, The First Affiliated Hospital of Fujian Medical University, Fuzhou, China

${ }^{2}$ Department of Neurosurgery, Fujian Neuromedicine Center, Fuzhou, China

Correspondence to Dr Dezhi Kang; kdz99988@vip.sina.com

\section{ABSTRACT}

Introduction The treatment decision and long-term outcomes of previously untreated cerebral cavernous malformation (U-CCM) are still controversial. Therefore, we are conducting a nationwide multicentre prospective registry study in China to determine the natural history and effect of surgical treatment on long-term outcomes in Chinese people with U-CCM.

Methods and analysis This study was started on 1 January 2018 and is currently ongoing. It is a cohort follow-up study across a 5-year period. Patients will be followed up for at least 3 years after inception. Patients with U-CCM will be enrolled from 24 Grade III, level A hospitals distributed all over China. The cohort size is estimated to be 1200 patients. Patients are registered in surgically treated group and conservatively treated group. Clinical characteristics, radiology information and laboratory data are prospectively collected using an electronic case report form through an electronic data capture system. The primary outcome of this study is poor clinical outcome at the last follow-up (modified Rankin Scale score $>2$ lasting at least 1 year). The secondary outcome includes symptomatic haemorrhage, drug refractory epilepsy, focal neurological deficits, morbidity and all-cause mortality during follow-up. Univariate and multivariate regression analysis will be performed to determine the risk factors for poor outcomes in all patients, and to estimate the effect of surgery. Life tables, Kaplan-Meier estimates, log-rank test and proportional hazards Cox regression will be used to analyse the followup data of conservatively treated patients to determine the natural history of U-CCM. Initial presentation and location of U-CCM are prespecified subgroup factors.

Ethics and dissemination The study protocol and informed consent form have been reviewed and approved by the Research Ethical Committee of First Affiliated Hospital of Fujian Medical University (FAHFMU-2018-003)

Written informed consent will be obtained from each adult participant or from the guardian of each paediatric participant. The final results will be published in peerreviewed journals.

Trial registration number NCT03467295.
Strengths and limitations of this study

- This is a multicentre (24 Grade III, level A hospitals) and large sample size (more than 1000 patients) prospective observational cohort study on previously untreated cerebral cavernous malformation (U-CCM) in China.

- This study will offer level B evidence for the controversial clinical questions about the treatment decision-making of U-CCM and determine the natural history of U-CCM in conservatively treated patients.

- The evidence level of this cohort study is not as high as a randomised controlled trial.

- The follow-up is relatively short for patients (less than 5 years) with incident CCM.

This cohort study only focuses on CCM, but does not include spinal cavernous malformations.

\section{INTRODUCTION}

Cerebral cavernous malformations (CCM) is the second most common cerebral vascular malformation. CCM affects people at any age and occurs throughout the central nervous system. ${ }^{1}$ The prevalence is estimated at $0.16 \%-0.5 \%$, with an annual detection rate estimated at $0.56 / 100000 /$ year for adults. ${ }^{23}$ Due to weakening of the vascular endothelial cell junctions or changes in lumen polarity, the typical histological presentations of CCM are multiple lumen malformations, vascular leakage at the brain capillary level and disruption of the blood-brain barrier. These pathological changes could cause haemorrhage, headache, seizures and focal neurological deficits (FND). For intracranial haemorrhage or FND, not including epileptic seizure, the 5-year risk of a first event is about $9.3 \%$, but the risk of recurrence dramatically increases to $42.4 \%$ in patients with CCM. ${ }^{4}$ The average time of rehemorrhage is only 10.5 months. ${ }^{5}$ Because 


\begin{tabular}{ll}
\hline Table 1 & The modified Rankin Scale \\
\hline Grade & Description \\
\hline 0 & $\begin{array}{l}\text { No symptoms at all } \\
1\end{array}$ \\
$\begin{array}{l}\text { No significant disability despite symptoms: able to } \\
\text { carry out all usual duties and activities }\end{array}$ \\
$\begin{array}{l}\text { Slight disability: unable to carry out all previous } \\
\text { activities but able to look after own affairs without } \\
\text { assistance }\end{array}$ \\
$\begin{array}{l}\text { Moderate disability: requiring some help, but able to } \\
\text { walk without assistance }\end{array}$ \\
$\begin{array}{l}\text { Moderately severe disability: unable to walk without } \\
\text { assistance and unable to attend to own bodily needs } \\
\text { without assistance }\end{array}$ \\
$\begin{array}{l}\text { Severe disability: bedridden, incontinent and requiring } \\
\text { constant nursing care and attention }\end{array}$ \\
\hline
\end{tabular}

of these risks, microsurgical excision is often used to treat CCM. However, despite decades of neurosurgical experience in this field, evidence supporting surgical resection of CCM remains conflicting. ${ }^{1}$ The conflicting evidence is partly attributable to the shortage of evidence supporting CGM surgical treatment. To address the absence of highlevel evidence to support untreated CCM (U-CCM) treatment decisions, there is a need for a large sample size study of U-CCM treatment with a prospective concurrent group. China has the largest population in the world and may have the most extensive and valuable clinical record of U-CCM. Therefore, we are conducting a nationwide multicentre prospective registry study in China to determine the natural history and effect of surgical treatment on long-term outcomes in Chinese people with U-CCM.

\section{Study objectives}

To determine whether surgical treatment improves the long-term outcomes of patients diagnosed with U-CCM, and to determine the natural history of U-CCM in
Chinese people based on conservative treatment arm. The long-term outcomes include modified Rankin Scale (mRS) score (table 1), morbidity and all-cause mortality. The natural history is studied from the incidence rate of symptomatic haemorrhage, drug refractory epilepsy and FND.

\section{METHODS AND ANALYSIS Study design}

This study is a nationwide multicentre prospective cohort study initiated by the Neurosurgery Department of First Affiliated Hospital of Fujian Medical University and supported by the National Cerebrovascular and Nervous System Difficult Diseases Diagnosis and Treatment Capacity Improvement Project (principal investigator: Dezhi Kang), with a grant from the National Development and Reform Commission, and National Health and Family Planning Commission, funding by the Fujian Province High level Neuromedical Center Construction Fund (principal investigator: Dezhi Kang) and a grant from the Government of Fujian Province (Grant number: HLNCCFJFY-003). This study began on 1 January 2018, and is currently ongoing. All eligible patients with U-CCM will be enrolled from 24 Grade III, level A hospitals distributed all over China (figure 1, table 2). This is a cohort follow-up study across a 5-year period. The integrity, accuracy and validity of the clinical data are supervised by the Clinical Research Data Security Committee of Fujian Medical University. The study design is presented in figure 2.

\section{Participants and study settings Patients}

All patients in this study should meet the inclusion and exclusion criteria. Written informed consent should be obtained from eligible adult patients or from the
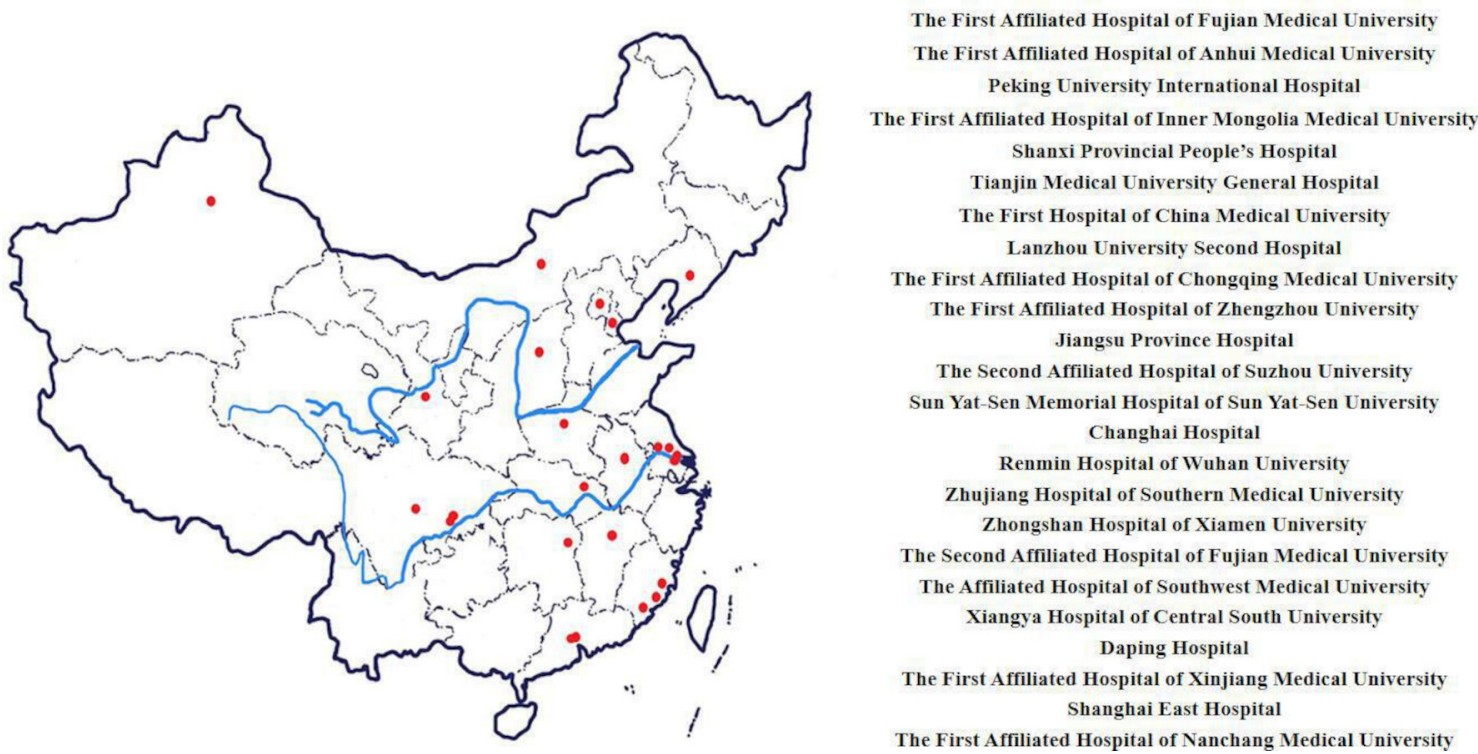

Figure 1 The geographical location and full names of the 24 subcentres of this study. 


\begin{tabular}{|c|c|c|}
\hline No. & Participating units & Province \\
\hline 1 & $\begin{array}{l}\text { The First Affiliated Hospital of Fujian } \\
\text { Medical University }\end{array}$ & Fujian \\
\hline 2 & $\begin{array}{l}\text { The First Affiliated Hospital of Anhui } \\
\text { Medical University }\end{array}$ & Anhui \\
\hline 3 & $\begin{array}{l}\text { Peking University International } \\
\text { Hospital }\end{array}$ & Beijing \\
\hline 4 & $\begin{array}{l}\text { The First Affiliated Hospital of Inner } \\
\text { Mongolia Medical University }\end{array}$ & Inner Mongolia \\
\hline 5 & Shanxi Provincial People's Hospital & Shanxi \\
\hline 6 & $\begin{array}{l}\text { Tianjin Medical University General } \\
\text { Hospital }\end{array}$ & Tianjin \\
\hline 7 & $\begin{array}{l}\text { The First Hospital of China Medical } \\
\text { University }\end{array}$ & Liaoning \\
\hline 8 & Lanzhou University Second Hospital & Lanzhou \\
\hline 9 & $\begin{array}{l}\text { The First Affiliated Hospital of } \\
\text { Chongqing Medical University }\end{array}$ & Chongqing \\
\hline 10 & $\begin{array}{l}\text { The First Affiliated Hospital of } \\
\text { Zhengzhou University }\end{array}$ & Zhengzhou \\
\hline 11 & Jiangsu Province Hospital & Jiangsu \\
\hline 12 & $\begin{array}{l}\text { The Second Affiliated Hospital of } \\
\text { Suzhou University }\end{array}$ & Suzhou \\
\hline 13 & $\begin{array}{l}\text { Sun Yat-sen Memorial Hospital of } \\
\text { Sun Yat-sen University }\end{array}$ & Guangdong \\
\hline 14 & Changhai Hospital & Shanghai \\
\hline 15 & $\begin{array}{l}\text { Renmin Hospital of Wuhan } \\
\text { University }\end{array}$ & Wuhan \\
\hline 16 & $\begin{array}{l}\text { Zhujiang Hospital of Southern } \\
\text { Medical University }\end{array}$ & Guangdong \\
\hline 17 & $\begin{array}{l}\text { Zhongshan Hospital of Xiamen } \\
\text { University }\end{array}$ & Fujian \\
\hline 18 & $\begin{array}{l}\text { The Second Affiliated Hospital of } \\
\text { Fujian Medical University }\end{array}$ & Fujian \\
\hline 19 & $\begin{array}{l}\text { The Affiliated Hospital of Southwest } \\
\text { Medical University }\end{array}$ & Sichuan \\
\hline 20 & $\begin{array}{l}\text { Xiangya Hospital of Central South } \\
\text { University }\end{array}$ & Hunan \\
\hline 21 & Daping Hospital & Chongqing \\
\hline 22 & $\begin{array}{l}\text { The First Affiliated Hospital of } \\
\text { Xinjiang Medical University }\end{array}$ & Xinjiang \\
\hline 23 & Shanghai East Hospital & Shanghai \\
\hline 24 & $\begin{array}{l}\text { The First Affiliated Hospital of } \\
\text { Nanchang Medical University }\end{array}$ & Nanchang \\
\hline
\end{tabular}

guardians of eligible paediatric patients. All patients in this prospective study can withdraw at any time. The inclusion and exclusion criteria are as follows: inclusion criteria: (1) definite diagnosis of CCM on the basis of brain MRI (T1, T2, SWI and T2-fluid-attenuated inversion recovery) or pathological examination in surgically treated patients; (2) patients without any previous invasive therapy (microsurgery, radiosurgery or multimodality treatment); (3) without neurological deficits (mRS score
0-1) before enrolment and (4) informed consent, and willing to accept long-term follow-up. Exclusion criteria included: (1) patients receiving emergency surgery due to acute intracranial haemorrhage; (2) patients with other intracranial diseases, such as aneurysms, tumours or other vascular malformations, except developmental venous anomalies (DVA); (3) patients with severe underlying disease, which affects their functional status and short-term life expectancy and (4) patients with severe mental or psychological disease.

\section{Data collection}

Clinical data, radiological information and laboratory data are prospectively collected through electronic case report form (eCRF) and uploaded online by each participating neurosurgery centre to form the prospective clinical database in the First Affiliated Hospital of Fujian Medical University. A third-party operational research consultant company (Real Data Medical Research) will manage, audit and lock the database. Clinical data includes demographic information, medical history, family history, initial presentation, physical examination, mRS score and treatment information. CCM location, CCM size (T2 sequence, excluding the haemosiderin ring), haematoma size (CT scans), associated DVA and multiplicity are documented. All patients are assessed according to the reporting standards of CCM research. ${ }^{6}$ Laboratory data includes blood routine, serum biochemical, coagulation tests, liver and kidney function tests, $\mathrm{C}$ reactive protein, procalcitonin and some other uncommon blood biomarkers, such as interleukin (IL)-6, matrix metalloproteinase (MMP)-2, MMP-9, vascular endothelial growth factor, IL-2, immunoglobulins, tumour necrosis factor- $\alpha$ and IL-1 $\beta$. All the clinical data, radiological information, laboratory data and blood samples are collected at inception point and on admission. The clinical coordinators (CRC) in each centre are responsible for collecting all the data needed in eCRF. The CRCs were trained before the start of this study.

\section{Treatment methods}

On admission, all patients are counselled regarding potential advantages and risks for different treatments of different kinds of CCM. Surgical and conservative treatments are both proposed as first-line therapeutic options according to the latest guideline from the US Angioma Alliance for symptomatic lesions and asymptomatic but accessible lesions. ${ }^{2}$ After time for consideration, the consultant and the patient jointly decide on the treatment method. Patients are registered in the initial surgery group and conservative treatment group (CTG) based on the treatment.

\section{Surgical treatment}

All patients undergo routine preoperative CT and MRI examination. Functional MRI, including sagittal threedimensional T1, blood-oxygen-level-dependent functional MRI and diffusion tensor imaging (DTI) are 


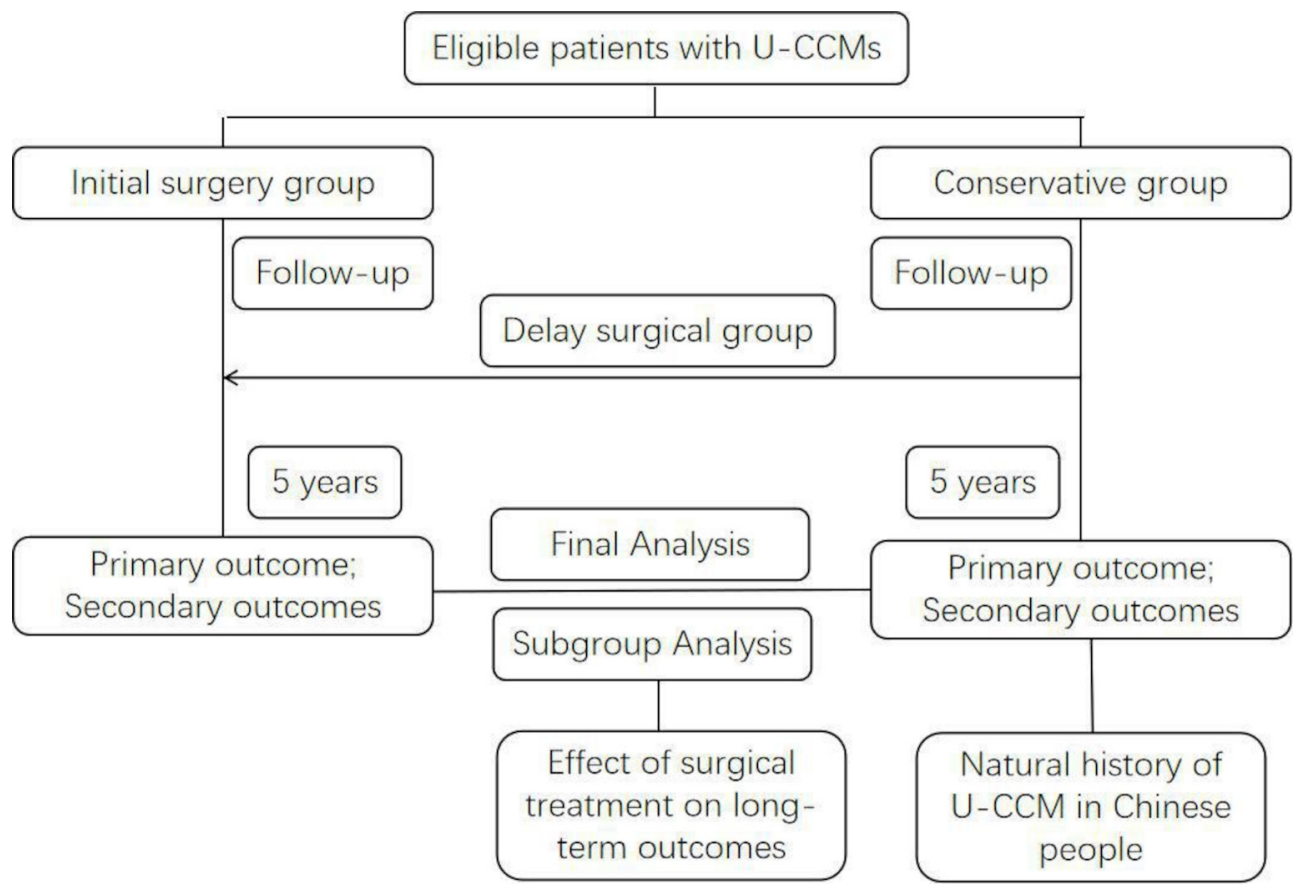

Figure 2 Diagram of the study protocol. U-CCM, untreated cerebral cavernous malformation.

suggested for patients with eloquent lesions. Surgical approaches are designed according to preoperative radiological images. The key points for surgery are as follows: less transgression of normal brain tissue; identification and protection of eloquent brain tissue; sparing associated DVA; haemosiderin rim resection for noneloquent and superficial CCM, and sparing yellowish tissue for eloquent and deep ones; extended resections (mesial resection and standard temporal lobe resection) are recommend for epileptogenic CCM based on intraoperative electroencephalogram (EEG). Postoperative CT and pre-discharge MRI are obtained to detect the perioperative complications and residual of CCM. All image sets are uploaded to the data centre via the internet. Two neuroradiologists and one experienced neurosurgeon read the images independently to verify and document the complications and CCM residual. After surgery, antiepilepsy drugs (AEDs) are started and withdrawn under the supervision of the attending neurologist (Yuanxiang Lin) according to a standardised algorithm. In general, for patients with seizure before surgery, AEDs are continued on the day of the surgery and withdrawn in case they are seizure-free for at least 1 year and show normal EEG scalp recordings. Radiosurgery is only recommended for patients with unresectable lesions, which is out of the scope of this study.

\section{Conservative treatment}

For CTG patients, we give the best medicine and supportive treatment, such as AEDs, analgesic and neurotropic drugs. For patients with underlying diseases such as hypertension, hyperlipidaemia or diabetes mellitus, after consulting with the relevant experts, they are treated accordingly. We prospectively record the drug administration and disease development. During the observation period, if patients suffer from cerebral haemorrhage or deterioration of associated symptoms, surgical treatment can be performed. However, following this, the observation is ended, and the patient is shifted to the delay surgical treatment group.

\section{Ethics and dissemination}

The study protocol and informed consent form (V2.0 2018-3-1) have been reviewed and approved by the Research Ethical Committee of First Affiliated Hospital of Fujian Medical University (FAHFMU-2018-003) and by research ethical committee of each participating centres. This study will be conducted in accordance with the ethical principles of the Declaration of Helsinki and the good clinical practice guidelines of the International Conference on Harmonisation. Written informed consent will be obtained from each adult participant or from the guardian of each paediatric participant. Dezhi Kang is the supervisor of this cohort study. The interim and final results will be published in peer-reviewed journals.

\section{Follow-up}

This is a cohort follow-up study across a 5-year period with a 2-year interval of enrolment. Patients will be followed up for at least 3 years after inception. The inception point for the conservatively managed group is the date of medical consultation that leads to U-CCM diagnosis. The inception point for the surgically treated group is the date of the first surgical treatment. Patient outcome will be assessed by a neurosurgeon or a nurse clinician from each participating centre. All the assessors are blinded to the treatment methods and have been professionally trained in evaluating patient outcomes (including using 
the mRS score) before this study began. Outcomes will be prospectively documented through telephone or patient's routine clinical visit every 6 months in the first follow-up year and annually later. The outcomes of patients with CCM will be recorded in the particular follow-up period, given that the number of patients is high and the pathological progress of CCM is slow. Once patients with CCM suffer haemorrhage, seizure, FND or deteriorating condition, the downward mRS score $(<2)$ will reveal the poor outcomes. Important radiological and clinical information, and data will be collected and uploaded by our electronic data capture (EDC) system.

\section{Outcomes}

Patient outcome will be assessed by a trained neurosurgeon or a nurse clinician from each of the participating centres. Symptomatic haemorrhage and FND during prospective follow-up are defined according to the current guidelines of the Angioma Alliance Scientific Advisory Board. ${ }^{6}$ CCM haemorrhage is standardised as 'requiring acute or subacute onset of symptoms (any of headache, epileptic seizure, impaired consciousness or new/worsened FND referable to the anatomic location of the CCM) accompanied by radiological, pathological, surgical or, rarely, only cerebrospinal fluid evidence of recent extralesional or intralesional haemorrhage'. ${ }^{6}$ FND is defined as new or worsened neurological deficit referable to the CCM anatomical location with or without timely investigation to rule out evidence of recent haemorrhage. ${ }^{7}$ 'Drug-resistant epilepsy' is defined as failure of adequate trials of two tolerated, appropriately chosen and used AED schedules (whether as monotherapies or in combination) to achieve sustained seizure freedom. ${ }^{8}$ Morbidity is defined as a persistent ( $>12$ months) decrease of a minimum of 1 point on the $\mathrm{mRS}$ score compared with the score at inception or on admission. Poor outcome is defined as a final $\mathrm{mRS}>2$ lasting at least 1 year at the last follow-up.

\section{Primary outcome}

The primary outcome of this study is poor clinical outcome at the last follow-up ( $\mathrm{mRS}>2$ lasting at least 1 year).

\section{Secondary outcomes}

Secondary outcomes include symptomatic haemorrhage, drug refractory epilepsy, FND, morbidity and all-cause mortality.

Secondary outcomes include symptomatic haemorrhage, drug refractory epilepsy, FND, morbidity and allcause mortality.

\section{Expected sample size}

Before the start of this study, we conducted a survey through a questionnaire to ascertain the number of patients with CCM treated or consulted at each participating centre. According to the survey results, each centre is expected to enrol more than 25 patients a year on average. There are 24 participating centres in total.
Thus, each year, we will enrol more than 600 patients. With a 2-year interval of enrolment, we expect to register 1200 patients and follow-up for at least 3 years. As this is a prospective cohort registration study, the actual sample size depends on the number of patients enrolled in during this study period. We will enrol every patient with U-CCM who meets the inclusion and exclusion criteria to accumulate sufficient numbers of primary and secondary outcomes to enable us to analyse our potential predictors in statistical analyses.

\section{Data management}

All data will be prospectively collected using an eCRF through an EDC system (Real Data eClinical Research System). Each participating neurological centre is assigned an account, and can access and upload patient information through a login with a password. Data safety, data quality, data auditing and database locking will be managed by a third party (Real Data Medical Research), who will be responsible for notifying any issues that may arise during the whole cohort study. The entire study will be supervised by the Research Ethical Committee of First Affiliated Hospital of Fujian Medical University and Clinical Research Data Security Committee of Fujian Medical University. Any issue occurring during the cohort study will be reported to these two oversight authorities. Recommendations from these two authorities will be forwarded to the principal investigator to balance the risk and benefit. The oversight authorities have the right to terminate the study if great risk occurs during the study.

\section{Statistical analysis}

Baseline characteristics: Initial presentation and location of U-CCM are prespecified subgroup factors. U-CCM location is documented as brainstem, cerebellar, thalamus, basal ganglia, cortex, and subcortex. We dichotomize location as deep (brainstem, thalamus and basal ganglia) versus superficial (cortex, subcortex of cerebrum and cerebellum) for analyses. If a patient has multiple CCM, we document the locations of the CCM that is resected or the symptomatic CCM if treated conservatively; in asymptomatic adults, deep CCM locations take precedence because it is a predictor of interest. We dichotomise initial presentation into hemorrhagic and nonhemorrhagi for analyses. If following initial presentatio a clinical event occure that lea to surgery of $\mathrm{U}-\mathrm{CCM}$, this event is recorded as the presentation in the treated group to reflect the indication for treatmentNatu historThe natu history of U-CCM isanalyze inconservative treatment armpatient. The prospective $\mathrm{mR}$, symptomatichemorrha, drug refractory epilepsy, FND, morbidity and all-cause mortality rate are determined based on the number of the events during the follow-up period divided by the number of prospective person-years of follow-up (an incidence rate); the annual rates are presented as per cent per person-year. The life tables and Kaplan-Meier estimates will be used to analyse the follow-up data of prospective symptomatic haemorrhage, drug refractory 
epilepsy and FND. For patients who are event-free at presentation, survival analyses of time to first event starts at the date of initial presentation and stops at the date of the first outcome event or the date of censoring. For patients presenting with the symptoms, or the symptoms beginning during follow-up, survival analyses of time to earliest recurrent event starts at the date of the first event and stops at the date of the earliest recurrent outcome or the date of censoring. We censor follow-up at the earliest occurrence of any of the following: death unrelated to CCM, first CCM surgical or radiological treatment, losing follow-up or at last clinical visit. Univariate comparisons with the log-rank test and multivariate comparisons with Cox regression will be performed to detect the risk factors independently associated with the concerned events. We plan to build predictive models for prospective symptomatic haemorrhage, drug refractory epilepsy and FND after diagnosis on the basis of the findings of the multivariable comparisons with Cox regression. We will use the data of the first half of the conservatively treated patients to develop the models and then the second half of patients as a test set.

Baseline characteristics: Initial presentation and location of U-CCM are prespecified subgroup factors. U-CCM location is documented as brainstem, cerebellar, thalamus, basal ganglia, cortex, and subcortex. We dichotomize location as deep (brainstem, thalamus and basal ganglia) versus superficial (cortex, subcortex of cerebrum and cerebellum) for analyses. If a patient has multiple CCM, we document the locations of the CCM that is treated or the symptomatic CCM if treated conservatively; in asymptomatic adults, deep CCM locations take precedence because it is a predictor of interest. We dichotomise initial presentation into hemorrhagic and nonhemorrhagi for analyses. If following initial presentatio a clinical event occure that lea to surgery of U-CCM, this event is recorded as the presentation in the treated group to reflect the indication for treatmentNatu historThe natu history of U-CCM isanalyze inconservative treatment armpatient. The prospective $\mathrm{mR}$, symptomatichemorrha, drug refractory epilepsy, FND, morbidity and all-cause mortality rate are determined based on the number of the events during the follow-up period divided by the number of prospective person-years of follow-up (an incidence rate); the annual rates are presented as per cent per person-year. The life tables and Kaplan-Meier estimates will be used to analyse the follow-up data of prospective symptomatic haemorrhage, drug refractory epilepsy and FND. For patients who are event-free at presentation, survival analyses of time to first event starts at the date of initial presentation and stops at the date of the first outcome event or the date of censoring. For patients presenting with the symptoms, or the symptoms beginning during follow-up, survival analyses of time to earliest recurrent event starts at the date of the first event and stops at the date of the earliest recurrent outcome or the date of censoring. We censor follow-up at the earliest occurrence of any of the following: death unrelated to
CCM, first CCM surgical or radiological treatment, losing follow-up or at last clinical visit. Univariate comparisons with the log-rank test and multivariate comparisons with Cox regression will be performed to detect the risk factors independently associated with the concerned events. We plan to build predictive models for prospective symptomatic haemorrhage, drug refractory epilepsy and FND after diagnosis on the basis of the findings of the multivariable comparisons with Cox regression. We will use the data of the first half of the conservatively treated patients to develop the models and then the second half of patients as a test set.

Effect of surgical treatment: The long-term outcomes will be compared between the CTG and the surgical treatment group. The mRS score is the main parameter for evaluating the prognosis. Parametric and non-parametric statistics will be used for between-group comparisons. We used OR, 95\% CI for categorical variables. Associations of variables and primary outcome (mRS score at last follow-up) will be identified using a univariate and multivariate regression analysis. In multivariable analyses, we will adjust the OR of surgical treatment for covariates that differ between the groups at baseline, or which may influence CCM outcome (location of U-CCM and mode of initial presentation). For more accurate estimation, propensity score matching could be used in subgroup analyses. Statistical analyses will be performed using the Statistical Package for the Social Sciences software (V.24.0; SPSS).

\section{Patient and public involvement}

The research questions were designed by the multidisciplinary team of CCM in our centre based on clinical practice and literature review, and audited by the Clinical Research Centre of The First Affiliated Hospital of Fujian Medical University. Neither patients nor the public were directly involved in the selection of outcome measures, design and implementation of the study. Patients will be informed of the study flow and provided feedback on reducing burden. The main results of the study will be disseminated to participants who are interested in their results from baseline and end-of-study assessments.

\section{DISCUSSION}

In the clinical practice, deciding on conservative management or surgical intervention for CCM remains difficult and controversial. ${ }^{12}$ In the literature, the authors could not identify high-quality studies that show dramatic benefit or harm from surgery. ${ }^{9}{ }^{10}$ Only a few studies show beneficial effects of surgical resection on seizures caused by CCM. ${ }^{11}{ }^{12}$ In 2014, a non-randomised population-based study compared surgical excision to conservative management and revealed that CCM excision was associated with worse outcomes over 5 years compared with conservative management. ${ }^{13}$ However, we know that microsurgical resection can relieve the mass effect associated with haemorrhage and interrupt the cycle of repeat haemorrhage 
and progressive neurological decline. ${ }^{14}$ Furthermore, the widespread use of modern state-of-the-art techniques, including functional MRI, DTI, neuronavigation and neuromonitoring, renders surgical resection of CCM safer than before, even in critical areas. ${ }^{1516}$ In our previous studies, we surgically treated CCM involving the posterior limb of internal capsule and corticospinal tract with acceptable risks and long-term functional outcomes. ${ }^{17-19}$ Thus, the latest published guideline for the clinical management of CCM by the Angioma Alliance Scientific Advisory Board Clinical Experts Panel recommended surgery in the following subgroup of patients: (a) surgical resection may be considered in solitary asymptomatic CCM if easily accessible in non-eloquent area (class IIb, level C); (b) surgery may be considered in symptomatic, easily accessible CCM, with mortality and morbidity equivalent to living with the CCM for about 2 years (class IIb, level B); and (c) surgical resection may be considered in deep CCM if symptomatic or after prior haemorrhage, with mortality and morbidity equivalent to living with the CCM for 5-10 years (class IIb, level B). ${ }^{2}$ However, most of evidences supporting the recommendations are based on the retrospective studies or the single centre studies with a small sample size and most of the studies are deemed to be biassed. ${ }^{2}$ China has a large reservoir of CCM, affecting $0.16 \%-0.5 \%$ of the population. ${ }^{3}$ Therefore, we are conducting a nationwide multicentre prospective registry study with a large sample size in China to address the absence of high-level evidence to support U-CCM treatment decisions.

Despite the availability of microsurgical excision for CCM treatment and known genetic causes of most familial forms of CCM, uncertainties remain about the cause, diagnosis, prognosis, treatment and care. ${ }^{1}$ To prioritise these uncertainties about CCM for researchers, in 2016, the James Lind Alliance Priority Setting Partnerships proposed the top 10 research priorities for cavernous malformations. ${ }^{1}$ The objective of this study is to answer the first and the most important question of the top 10 research priorities. The questions of 3, 6 and 9 are contained in the objective of natural history study, developing predictive models for the prospective symptomatic haemorrhage and drug refractory epilepsy in this study respectively. We believe that the results of this nationwide multicentre prospective concurrent cohort study with a large sample size, will answer these questions to a certain extent. Expectantly, this study will offer level B evidence for clinical practice to manage CCM and provide the preconditions for following randomised controlled trails.

\section{Strengths and limitations}

There are several strengths in our study. First, this is a multicentre prospective cohort study on CCM in China with a large sample size with 24 hospitals participating from all over China. It can provide evidence which is both relatively objective and consistent with the realworld treatment of CCM in China. Second, the clinical study is easier to carry out due to the concise process and the standard operating procedures. However, some limitations of this study are as follows: (1) the evidence level of this cohort study is not as high as a randomised controlled trial and (2) the follow-up is relatively short for incident patients with CCM. Third, we only focus on CCM but not on spinal cavernous malformations, which is limited by various levels of diagnosis and treatment in different medical centres.

Acknowledgements Thanks for the technical support from Real Data Medical Research to design the electronic data capture system and to manage the data. Xiaoxia Jiang, Ying Fu, Jiaheng Xu, Xiaoyong Chen and Ang Li participated in the work on fund application, study design, clinical reception, evaluation, management and operation.

Contributors DK has obtained research funding and is the principal investigator of the study. FL, QH, ZG and DK have developed this study protocol, including ensuring ethical principal, designing study methodology, and drafting and revising the manuscript. LY, DW, SZ and YL have provided helpful feedback for all aspects of the work and participated in the final design of the study.

Funding This study is funded by the National Cerebrovascular and Nervous System Difficult Diseases Diagnosis and Treatment Capacity Improvement Project (principal investigator: DK), a grant from the National Development and Reform Commission and National Health and Family Planning Commission, and supported by the Fujian Province High level Neuromedical Center Construction Fund (principal investigator: DK), a grant from the Government of Fujian Province (Grant number: HLNCCFJFY-003). The funding bodies support the employment of contract research organisation (Real Data Medical Research) and all of expenses during research.

Map disclaimer The depiction of boundaries on the map(s) in this article does not imply the expression of any opinion whatsoever on the part of BMJ (or any member of its group) concerning the legal status of any country, territory, jurisdiction or area or of its authorities. The map(s) are provided without any warranty of any kind, either express or implied.

\section{Competing interests None declared.}

Patient and public involvement The research questions were designed by the multidisciplinary team of CCM in our centre based on clinical practice and literature review, and audited by the Clinical Research Centre of The First Affiliated Hospital of Fujian Medical University. Neither patients nor the public were directly involved in the selection of outcome measures, design and implementation of the study. Patients will be informed of the study flow and provided feedback on reducing burden. The main results of the study will be disseminated to participants who are interested in their results from baseline and end-of-study assessments.

Patient consent for publication Obtained.

Provenance and peer review Not commissioned; externally peer-reviewed.

Open access This is an open access article distributed in accordance with the Creative Commons Attribution Non Commercial (CC BY-NC 4.0) license, which permits others to distribute, remix, adapt, build upon this work non-commercially, and license their derivative works on different terms, provided the original work is properly cited, appropriate credit is given, any changes made indicated, and the use is non-commercial. See: http://creativecommons.org/licenses/by-nc/4.0/.

ORCID iD

Dezhi Kang http://orcid.org/0000-0002-0906-9456

\section{REFERENCES}

1 Al-Shahi Salman R, Kitchen N, Thomson J, et al. Top ten research priorities for brain and spine cavernous malformations. Lancet Neurol 2016;15:354-5.

2 Akers A, Al-Shahi Salman R, A Awad I, et al. Synopsis of guidelines for the clinical management of cerebral cavernous malformations: consensus recommendations based on systematic literature review by the angioma alliance scientific Advisory board clinical experts panel. Neurosurgery 2017;80:665-80.

3 Morris Z, Whiteley WN, Longstreth WT, et al. Incidental findings on brain magnetic resonance imaging: systematic review and metaanalysis. BMJ 2009;339:b3016.

4 Al-Shahi Salman R, Hall JM, Horne MA, et al. Untreated clinical course of cerebral cavernous malformations: a prospective, population-based cohort study. Lancet Neurol 2012;11:217-24. 
5 Horne MA, Flemming KD, Su I-C, et al. Clinical course of untreated cerebral cavernous malformations: a meta-analysis of individual patient data. Lancet Neurol 2016;15:166-73.

6 Al-Shahi Salman R, Berg MJ, Morrison L, et al. Hemorrhage from cavernous malformations of the brain: definition and reporting standards. angioma alliance scientific Advisory board. Stroke 2008;39:3222-30.

7 Zhang L, Yang W, Jia W, et al. Comparison of outcome between surgical and conservative management of symptomatic spinal cord cavernous malformations. Neurosurgery 2016;78:552-61.

8 Kwan P, Arzimanoglou A, Berg AT, et al. Definition of drug resistant epilepsy: consensus proposal by the AD hoc Task force of the ILAE Commission on therapeutic strategies. Epilepsia 2010;51:1069-77.

9 Poorthuis M, Samarasekera N, Kontoh K, et al. Comparative studies of the diagnosis and treatment of cerebral cavernous malformations in adults: systematic review. Acta Neurochir 2013;155:643-9.

10 Bicalho VC, Bergmann A, Domingues F, et al. Cerebral cavernous malformations: patient-reported outcome validates conservative management. Cerebrovasc Dis 2017;44:313-9.

11 Dammann P, Wrede K, Jabbarli R, et al. Outcome after conservative management or surgical treatment for new-onset epilepsy in cerebral cavernous malformation. J Neurosurg 2017;126:1303-11.

12 Lee $\mathrm{Y}$, Cho KH, Kim HI, et al. Clinical outcome following medical treatment of cavernous malformation related epilepsy. Seizure 2017;45:64-9.
13 Moultrie F, Horne MA, Josephson CB, et al. Outcome after surgical or conservative management of cerebral cavernous malformations. Neurology 2014;83:582-9.

14 Zaidi HA, Mooney MA, Levitt MR, et al. Impact of timing of intervention among 397 consecutively treated brainstem cavernous malformations. Neurosurgery 2017;81:620-6.

15 Garcia RM, Ivan ME, Lawton MT. Brainstem cavernous malformations: surgical results in 104 patients and a proposed grading system to predict neurological outcomes. Neurosurgery 2015;76:265-77.

16 Pandey P, Westbroek EM, Gooderham PA, et al. Cavernous malformation of brainstem, thalamus, and basal ganglia: a series of 176 patients. Neurosurgery 2013;72:573-89.

17 Li D, Jiao Y-M, Wang L, et al. Surgical outcome of motor deficits and neurological status in brainstem cavernous malformations based on preoperative diffusion tensor imaging: a prospective randomized clinical trial. J Neurosurg 2018;130:286-301.

18 Lin Y, Lin F, Kang D, et al. Supratentorial cavernous malformations adjacent to the corticospinal tract: surgical outcomes and predictive value of diffusion tensor imaging findings. J Neurosurg 2018;128:541-52.

19 Lin F, Wu J, Wang L, et al. Surgical treatment of cavernous malformations involving the posterior limb of the internal capsule: utility and predictive value of preoperative diffusion tensor imaging. World Neurosurg 2016;88:538-47. 